\title{
Hans Maier \\ Ein Provisorium hat sich bewährt. Das Grundgesetz nach 60 Jahren
}

Vor 60 Jahren, am 23. Mai 1949, wurde in Bonn das Grundgesetz verkündet. Es war als Provisorium und »Transitorium " (Theodor Heuss) gedacht - ausdrücklich vermied man das Wort Verfassung. Dem staatlichen Leben sollte »für eine Übergangszeit eine neue Ordnung « gegeben werden - so formulierte es die alte, 1990 neugefasste Präambel. Aber nichts ist bekanntlich so dauerhaft wie das Provisorische: Inzwischen hat das Grundgesetz - und mit ihm die Bundesrepublik Deutschland - bereits das stattliche Alter von 60 Jahren erreicht, fast das Vierfache der Jahre, die der Weimarer Republik gegönnt waren. Die Zweite Republik ist glücklicher, so scheint es, als die Erste. Sie lebt zwar nicht auf großem, aber auf solidem Fuß. Sie erfreut sich breiter Zustimmung in der Öffentlichkeit. Das Grundgesetz ist in sechzig Jahren aus einem Provisorium zur Verfassung aller Deutschen geworden. Es hat Deutschland als Verfassungsstaat geprägt. Die Bürger - wenigstens in ihrer ganz überwiegenden Mehrzahl - fühlen sich in dieser Verfassung gut aufgehoben. ${ }^{1}$ Sie respektieren sie mit Stolz, ja mit patriotischen Gefühlen. »Verfassungspatriotismus " nannte Dolf Sternberger das 1982 in einer Rede bei der 25-Jahr-Feier der Akademie für Politische Bildung Tutzing, und er erinnerte an einen alten, auch heute unverändert wahren Satz von La Bruyère: »Es gibt kein Vaterland in der Despotie. $\aleph^{2}$

\section{Geschlagen und befreit: Die Deutschen nach 1945}

Die meisten der heute lebenden Deutschen sind nach 1949 geboren. Die Zahl derer, die sich noch an die unmittelbare Kriegs- und Nachkriegszeit erinnern, an die Jahre, die dem Bonner Staatsakt von 1949 vorausgingen, nimmt ab. Die Nachkriegszeit - das war eine Zeit der Improvisationen, der Überlebenskünste; der lähmenden Erstarrung im großen und der flinken Beweglichkeit im kleinen. Auf der einen Seite die alltägliche Misere: Ruinen, Gedränge in zertrümmerten Häusern und Städten, Beschränkung aufs Nächste, Sorge ums Überleben, um die fällige Tagesration; eine Gesellschaft ohne Zukunftsaussichten, so schien es, Flüchtlinge, Heimkehrer, Ausgebombte, Wohnungssuchende Geschlagene mit einem Wort. Auf der anderen Seite große weltpolitische Bewegung

1 Vgl. Gary S. Schaal / Hans Vorländer / Claudia Ritzi, 60 Jabre Grundgesetz. Deutsche Identität im Spannungsfeld von Europäisierung. Ergebnisse einer repräsentativen Bevölkerungsbefragung, Dresden / Hamburg 2009.

2 Dolf Sternberger, »Verfassungspatriotismus « in: ders., Schriften, Band X: Verfassungspatriotismus, Frankfurt a.M. 1990, S. 3-16.

ZfP 56. Jg. 4/2009 
ringsherum: die Gründung der Vereinten Nationen, das Gericht der Sieger in Nürnberg, die angekündigte Verwandlung aller Machtpolitik in Moral, der verheißene Ewige Friede - aber auch der Katzenjammer des zerbröckelnden Kriegsbündnisses der Alliierten, versandende Konferenzen, beginnender Kalter Krieg.

Deutschland war 1945 durch die bedingungslose Kapitulation aus der Reihe der handelnden Mächte ausgeschieden. Der Kontrollrat der Alliierten hatte die Regierungsgewalt übernommen. Das Land war Objekt der Weltpolitik. Sein Schicksal stand zur Disposition. Deutschland war klein geworden, ein Land, in das Millionen von Menschen drängten, die Nahrung und Arbeit suchten. Viele Deutsche sahen damals im täglichen Kampf ums Überleben über die Vergangenheit hinweg. Gebannt vom eigenen Leid, bedachten sie selten das Leid, das anderen Völkern von Deutschen widerfahren war.

Doch die Deutschen hatten 1945 nicht nur den Krieg verloren, sie waren nicht nur in eine Katastrophe ohnegleichen gestürzt, die ihre Weiterexistenz als Volk und Staat in Frage stellte. Sie waren zugleich befreit worden: ${ }^{3}$ befreit von einem Regime, das sie aus eigener Kraft nicht abzuschütteln in der Lage waren, befreit durch Völker, die ihre Kriegsgegner waren und deren Sieg der bittere, aber notwendige Preis für das Ende der eigenen Unterdrückung war. Das ist eine Einsicht, die heute, nach 60 Jahren, wohl von der überwältigenden Mehrheit des deutschen Volkes geteilt wird. Nach 1945 war sie noch keineswegs Allgemeingut. Es bedurfte einiger Zeit, bis die Mehrheit der Deutschen die Chancen zu erkennen begannen, die sich in der Nachkriegszeit eröffneten. Nachwirkungen der nationalsozialistischen Zeit reichten noch ins erste Nachkriegsjahrzehnt hinein. Noch im Mai 1955, zehn Jahre nach Kriegsende, meinten 48 \% der Westdeutschen nach einer Umfrage des Instituts für Demoskopie Allensbach, »ohne den Krieg « wäre Hitler einer der größten deutschen Staatsmänner gewesen. ${ }^{4}$ Dass diese Zahl zwanzig Jahre später auf $38 \%$ zurückgegangen war und dass die Gegenmeinung, das Dritte Reich sei »in jedem Fall eine schlechte Sache « gewesen, stetig wuchs und im Dezember 1992 in der alten Bundesrepublik $64 \%$, in den neuen Bundesländern sogar $69 \%$ Zustimmung fand, eine Zweidrittelmehrheit also in ganz Deutschland - das zeigt zweierlei: dass die Abkehr vom Nationalsozialismus im Lauf der langen Nachkriegsgeschichte allmählich definitiv und unumkehrbar wurde, aber auch, dass dieser Vorgang langsam vor sich ging und viel Zeit in Anspruch nahm. ${ }^{5}$

Es wäre wohl noch weniger rasch in Gang gekommen, hätte sich nicht nach Kriegsende die weltpolitische Szenerie verändert. Schon am 12. Mai 1945 hatte Churchill in einem Telegramm an den amerikanischen Präsidenten Truman von einem »Eisernen Vorhang«

3 Theodor Heuss sah dies in der 10. Sitzung des Parlamentarischen Rates am 8. Mai 1949 als »die tragischste und fragwürdigste Paradoxie der Geschichte für jeden von uns. Warum denn? Weil wir erlöst und vernichtet in einem gewesen sind « (Parlamentarischer Rat, Stenographische Berichte, 10. Sitzung vom 8. Mai 1949, S. 210).

4 Elisabeth Noelle / Erich Peter Neumann (Hrsg.), Jabrbuch der öffentlichen Meinung 1947-1955, Allensbach 1956.

5 Edgar Piel, »Spuren der NS-Ideologie im Nachkriegsdeutschland « in: Heinrich Oberreuter / Jürgen Weber (Hrsg.), Freundliche Feinde. Die Alliierten und die Demokratiegründung in Deutschland, München 1996, S. 145-160. 
gesprochen, der vor der Front der sowjetischen Truppen niedergegangen sei. Die Wendung verbreitete sich rasch und gab einem ganzen Zeitalter den Namen. Der Kalte Krieg zwischen den östlichen und westlichen Alliierten gewann in den folgenden Jahren eine unerwartete Schärfe. Ganz Europa, vor allem aber Deutschland, war betroffen. 1948 wurde die Tschechoslowakei durch einen Staatsstreich kommunistisch, in Deutschland stellte der Alliierte Kontrollrat seine Tätigkeit ein, die Russen begannen Berlin zu blockieren. Das war die Zeit, in der man nicht nur in Deutschland, sondern in Westeuropa die bange Frage stellte, wann und wo die sowjetische Expansion denn enden werde.

Angesichts dieser kritischen Situation drängten die Westalliierten auf Stabilisierung zumindest in den Westzonen des besetzten Deutschlands. Dort hatten sich - nach Flucht und Vertreibung von Millionen - rund achtzig Prozent der früheren Bevölkerung des Deutschen Reiches gesammelt - ein Präjudiz für den Schwerpunkt der künftigen politischen Ordnung. So entstand auf westalliierter wie auf deutscher Seite der Plan, die westlichen Teile dessen, was von Deutschland übriggeblieben war, zu einer neuen staatlichen Organisation zusammenzufügen. Die Wirtschaftseinheit der Bi- und Trizone sollte in eine politische Einheit transformiert werden; die Deutschen sollten Zug um Zug ihre Souveränität zurückerhalten; aus den »Eingeborenen von Trizonesien«, wie sie der Volkswitz mit einiger Selbstironie nannte, sollten wieder Vollbürger werden - Bürger der Bundesrepublik Deutschland. ${ }^{6}$

Was folgte, erscheint uns im Rückblick als eine Konsequenz aus den neuen weltpolitischen Polbildungen. Die staatliche Einheit Deutschlands zerbrach: In seinem östlichen Teil wurde das Land unter russischer Hegemonie ${ }^{7}$ straff in das System des Ostblocks eingegliedert, im Westen entstand unter der schrittweise sich lockernden Aufsicht der USA, Großbritanniens, Frankreichs ein politisches Gebilde von wachsender Eigenständigkeit. Hier konnten die geschlagenen Deutschen ihre Chance als Befreite nutzen; hier konnten sie die neugewonnene Freiheit stabilisieren und ausbauen; hier konnten sie Anschluss gewinnen an die europäischen - und an die eigenen - Traditionen des Rechts, der Solidarität, der Demokratie. So gewann ihr Beispiel in kurzer Zeit Anziehungskraft auch für diejenigen Landsleute, die an der Ausarbeitung des Grundgesetzes nicht hatten mitwirken können. Die Geschichte der alten Bundesrepublik ist die Geschichte einer erfolgreichen politischen Stellvertretung über viele Jahre hin - von 1949 bis zum Jahr 1990, in dem sich die getrennten Wege der Nachkriegszeit wieder zu einem gemeinsamen Weg vereinigten. ${ }^{8}$

Das alles war, im Inneren wie im Äußeren, kein selbstverständlicher Prozess. Ohne die Hilfe der einstigen Gegner hätten sich die neuen Möglichkeiten im Westen kaum eröffnet. Der Wandel des Klimas nach 1945, der in so deutlichem Kontrast steht zu der

6 Wolfgang Benz, Von der Besatzungsherrschaft zur Bundesrepublik. Stationen einer Staatsgründung 1946-1949, Frankfurt a.M. 1991.

7 Gerhard Wettig, »Kontrastprogramm santifaschistisch demokratische Ordnung: Sowjetische Ziele und Konzepte« in: Heinrich Oberreuter / Jürgen Weber, aaO, S. 101-123.

8 Heinrich August Winkler, Der lange Weg nach Westen, Bd. 2: Deutsche Geschichte vom "Dritten Reich « bis zur Wiedervereinigung, München 2000; Hans-Ulrich Wehler, Deutsche Gesellschaftsgeschichte, Bd. 5, Bundesrepublik und DDR 1949-1990, München 2008. 
Verhärtung und Feindschaft nach 1918, hatte einen Grund gewiss in den geschilderten weltpolitischen Veränderungen und Handlungszwängen. Gegenüber dem sowjetischen Imperialismus musste sich der Westen auf seine freiheitlichen Traditionen besinnen. Das geschlagene Deutschland war in den Prozess europäischer und westlicher Reorganisation einzubeziehen. Es durfte kein machtpolitisches Vakuum im Herzen Europas entstehen. Aber die entscheidenden Anstöße gingen doch, wie meist in der Geschichte, von einzelnen aus: in Europa von einer Reihe von Politikern, die nach 1948 die Zusammenarbeit durch neue und ungewöhnliche Initiativen intensivierten: Schuman, De Gasperi, Adenauer, Spaak, Monnet. In einer Zeit der Not, des Zerfalls der Solidarität, der nationalen Egoismen erreichten sie durch entschlossene Kooperation, dass das geteilte Europa zumindest im Westen ein Stück seiner Handlungsfähigkeit zurück gewann. Das erforderte nicht nur Weitblick und Zähigkeit - es erforderte angesichts der allgemeinen politischen Unsicherheit auch Mut und visionäre Kraft.

\section{Akzente des politischen Neubeginns}

Der neue Anfang in den Jahren 1948/49 war nicht einfach. Im Parlamentarischen Rat prallten die Gegensätze gelegentlich heftig aufeinander. ${ }^{9}$ Die Hauptschwierigkeit für die Verfassungsgründer lag im offenkundigen Mangel an Modellen und an Vorbildern. Wo sollte, wo konnte man anknüpfen? Am wenigsten natürlich beim unmittelbar vorangegangenen Dritten Reich. Aber gewiss auch nicht einfach bei der Weimarer Republik. Denn hatte nicht die Schwäche Weimars den Präsidialstaat und später den Führerstaat förmlich angezogen? Musste man nicht die Zweite Republik gegen ein ähnliches Schicksal des Machtverfalls und der Selbstzerstörung schützen? Auch die Vorgänge in Ost- und Mitteleuropa, welche die Verfassungsberatungen begleiteten, waren eine bedrückende Lektion. Sie bestätigten, was im Jahr 1945 nur eine Minderheit der westlichen Politiker gesehen hatte: dass die Geschichte der Unfreiheit mit Hitlers Tod keineswegs zu Ende war.

Aus dem Fehlschlag der Ersten Republik zog die Zweite Republik ihre Lehren. Von Anfang an versuchten die Autoren des Grundgesetzes den neuen demokratischen Staat vom Stigma des Dritten Reiches, aber auch von den Schwächen Weimars zu befreien. ${ }^{10}$ Neue Akzente wurden gesetzt: ${ }^{11}$ im Verständnis des Rechtsstaats (1), in der Formulierung eines demokratischen Minimalkonsenses (2), im Bekenntnis zum parlamentarischen

9 Michael F. Feldkamp, Der Parlamentarische Rat 1948-1949. Die Entstehung des Grundgesetzes, überarbeitete Neuausgabe, Göttingen 2008.

10 Friedrich Karl Fromme, Von der Weimarer Verfassung zum Bonner Grundgesetz. Die verfassungspolitischen Folgerungen des Parlamentarischen Rates aus Weimarer Republik und nationalsozialistischer Diktatur, 2. Auflage, Tübingen 1962. Vgl. zum Folgenden auch Volker Otto, Das Staatsverständnis des Parlamentarischen Rates. Ein Beitrag zur Entstebungsgeschichte des Grundgesetzes für die Bundesrepublik Deutschland, Düsseldorf 1971.

11 Das gilt bereits für die Vorarbeiten des Herrenchiemseer Verfassungskonvents. Siehe Peter März / Heinrich Oberreuter (Hrsg.), Weichenstellung für Deutschland. Der Verfassungskonvent von Herrenchiemsee, München 1999. 
Regierungssystem (3), in der Stärkung der Exekutive (4). Damit knüpfte das Grundgesetz zugleich an ältere Traditionen deutscher Geschichte an: an die Tradition des imperium limitatum, des gelinden Regiments, der durch Rechtsbindung gedämpften Staatsgewalt; an den Föderalismus als die den Deutschen geläufige regionale Form der Gewaltenteilung; an woblfahrts- und sozialstaatliche Überlieferungen und nicht zuletzt an die initiierende und gestaltende Rolle von Regierung und Verwaltung.

(1) Nach der Nazi-Tyrannei, dem Unrechtsstaat schlechthin, entschloss man sich, Staat und Recht aufs engste zu verbinden, den Staat selbst auf das Recht zu gründen, wie es der süddeutsche Konstitutionalismus - Rotteck - mehr als hundert Jahre zuvor in programmatischer Radikalität gefordert hatte. Der Staat sollte zum Treuhänder seiner Bürger werden - zu ihrem Rechtsbeistand, ihrem »Rechtsanwalt «. Das bedeutete nicht nur, dass die Grundrechte in der Verfassung neue Bedeutung und konkrete Geltung erhielten und dass die rechtsprechende Gewalt auf allen Ebenen gestärkt wurde - es bedeutete vor allem, dass der Rechtsschutz im öffentlichen Recht erweitert wurde wie nie zuvor in der deutschen Verfassungsgeschichte.

Der Staat - früher eine gebietende Übermacht - wurde zum normalen Gegenüber, er wurde durchsichtig. Arkansphären und hoheitliche, den Gerichten unzugängliche Zonen verschwanden - ganz und ungeteilt wurde mit Art. 19 Abs. 4 Grundgesetz der Rechtsweg gegenüber Staatseingriffen eröffnet. Das hieß nichts anderes, als dass nunmehr - mit deutscher Grundsätzlichkeit - staatliche Gewalt sich rechtlich vor den Bürgern auszuweisen hatte. Die Bundesrepublik Deutschland wurde mit innerer Konsequenz zum Rechtsstaat, Rechtswegestaat, Justizstaat. Das Recht wurde zur politischen Substanz des Gemeinwesens.

Es ist von heute her gesehen nicht erstaunlich, dass dieses kühne Programm nicht bis zum letzten Rest verwirklicht werden konnte, dass manches davon später abgedämpft, begradigt, zurückgeholt werden musste. Erstaunlich ist aber, wieviel sich bewährt und gehalten hat, wieviel inzwischen ganz selbstverständlich in den politischen Konsens eingegangen ist. Dies gilt vor allem für die Ausgestaltung der Grundrechte. Hier hat man mit Recht von einer »kopernikanischen Wende « ${ }^{12}$ gesprochen. Fragte man früher zuerst nach den Grenzen der Grundrechte, so fragte man nun nach ihrem Inhalt: Die Grundrechte begannen das Grundgesetz zu regieren, sein Verständnis zu steuern - nicht umgekehrt. Theorie und Rechtsprechung dehnten die faktische Geltung der Grundrechte nach allen Seiten so weit aus, wie es unter den Bedingungen unserer enger zusammenrückenden Gesellschaft überhaupt nur denkbar und möglich war. Die formalen Sicherungen der Freiheit wurden verstärkt. Um der Freiheit willen wurde der Freiheitsspielraum des einzelnen bewusst weit gezogen, was bedeutete, dass man auch die Möglichkeit des Missbrauchs in Kauf nahm. Vor allem die Rechtsprechung der obersten Bundesgerichte über Menschenwürde, Persönlichkeitsentfaltung, Meinungsfreiheit und Eigentum war bemüht, den Bürgern einen unantastbaren Bereich menschlicher Freiheit zu sichern, welcher der Einwirkung des Staates entzogen war.

12 Klaus Stern, Das Staatsrecht der Bundesrepublik Deutschland, Bd. 1, 2. Auflage, München 1984, S. 416.

ZfP 56. Jg. 4/2009 
Den neuen Rang des Rechts gegenüber der Politik hat vor allem die Verfassungsgerichtsbarkeit offenbar gemacht. Sie ist in den sechzig Jahren der Geltung des Grundgesetzes immer stärker in die Rolle des »Hüters der Verfassung « hineingewachsen. So hat das Bundesverfassungsgericht nicht nur den Vorrang des Grundgesetzes in der Rechtsordnung befestigt und die Bedeutung der Grundrechte für die Gesetzgebung herausgearbeitet, es hat auch streitige politische Grundsatzfragen einer Lösung zugeführt und so die Verfassung weiterentwickelt. Den politischen Prozess begleitend und mitentscheidend, brachte das Bundesverfassungsgericht schon durch seine Existenz die Souveränität des Rechts gegenüber der Politik zum Ausdruck. ${ }^{13}$

Die Dominanz des Rechts im politischen Prozess hatte freilich auch ihre Probleme Probleme, die heute deutlicher hervortreten als im Jahr 1949. Wohl hat sich das Bundesverfassungsgericht in seiner Judikatur im allgemeinen zurückgehalten und den Eindruck zu vermeiden versucht, es wolle Dinge entscheiden, die ihrer Natur nach ins Parlament gehören. Aber es kam doch dazu, dass Gesetzgebung und Exekutive der Judikative manches auf die Richterbank schoben. ${ }^{14}$ In der Weimarer Republik sprachen Verfassungsjuristen von der » Legalitätsreserve « des Reichspräsidenten in politischen Krisen. Im Bonner Regierungssystem könnte man von einer "Streitentscheidungsreserve« des Bundesverfassungsgerichts sprechen. Das muss nicht, es kann aber zu einer Dämpfung der gesetzgeberischen Entschlusskraft, zu einem Ritardando im Streitaustrag führen. Statt rationale Gründe und Gegengründe zu bemühen, schwenken die Kontrahenten dann das Fähnlein der Verfassungswidrigkeit oder -konformität. Hinter dem pragmatischen politischen Alltag tut sich eine Szene der Rechtstheorie auf mit sehr deutschen Grundsätzlichkeiten und Verabsolutierungen.

Das muss man wohl in Kauf nehmen, wenn man den Staat so entschieden »unter das Recht«stellt, wie es das Grundgesetz getan hat. Gab es doch nach 1945 viele Gründe, dem Recht mehr zu trauen als der Politik. Wen wundert es, dass unter solchen Bedingungen die Zahl der Richter wuchs und das Recht sich in alle Lebensbereiche hinein ausdehnte - bis hin zum deutschen Unikum der gerichtlichen Nachprüfbarkeit von Schulnoten? Von der Wohltat der Rechtssicherheit bis zur Plage der Verrechtlichung war es dann oft nur ein kleiner Schritt. Und so stand neben der Genugtuung über die neue Qualität des Rechts im Bonner Grundgesetz von Anfang an auch die Klage über die Nebenfolgen dieser erfreulichen Entwicklung: die Schwerfälligkeit der Justiz, die Dauer der Verfahren, die fehlende Bürgernähe der Entscheidungen, die mangelnde Verständlichkeit der Gesetzessprache.

(2) War die deutlichere Ausprägung des Rechtsstaats eine Antwort auf den NS-Staat, so zielte das Bemühen, den Wertkonsens zu stärken, den »nichtkontroversen Sektor der

13 Jutta Limbach (Hrsg.), Das Bundesverfassungsgericht. Geschichte - Aufgabe - Rechtsprechung, Heidelberg 2000.

14 Uwe Wesel, Der Gang nach Karlsrube. Das Bundesverfassungsgericht in der Geschichte der Bundesrepublik, München 2004. 
Verfassung " (Ernst Fraenkel) zu festigen, auf die Schwächen der Weimarer Demokratie. ${ }^{15}$ Diese war bekanntlich wehrlos gewesen gegenüber ihren mächtigen Feinden auf der Rechten und auf der Linken. Sie verfügte über keine Schutzmechanismen gegen Selbstzerstörung. Geschützt war allein das formale Prinzip der Volkssouveränität. Kam das souveräne Volk zu dem Ergebnis, die Demokratie gehöre besser abgeschafft, so war dagegen nach damaligem Verfassungsverständnis kein Kraut gewachsen. Und so konnte auch die Machtergreifung einer totalitären Partei in wesentlichen Punkten hinter der Fassade demokratischer Legalität vor sich gehen.

Von solch prinzipiellem Relativismus nahm man im Grundgesetz entschlossen Abschied. An die Stelle eines positivistischen und formalistischen Demokratieverständnisses trat die freibeitliche demokratische Grundordnung - ein Minimalkonsens, geeignet, die Kämpfe und Konflikte einer pluralistischen Gesellschaft wirksam zu unterfangen. Dies war die Geburtsstunde der »wehrhaften « oder - wie andere sagten - der »wertbestimmten« Demokratie. Die freiheitliche demokratische Grundordnung wurde später vom Bundesverfassungsgericht in genaueren Bestimmungen konkretisiert als rechtsstaatliche Herrschaft auf der Grundlage der Selbstbestimmung des Volkes, nach dem Willen der jeweiligen Mehrheit, nach den Prinzipien der Freiheit und Gleichheit. ${ }^{16}$

Die totalitäre Bedrohung war in den fünfziger Jahren, als das Bundesverfassungsgericht seine Urteile gegen die SRP und die KPD sprach, noch in frischer Erinnerung. Sie ist inzwischen - so scheint es wenigstens - in eine größere Ferne gerückt. Freilich, Gefahren solcher Art können auch in verfassungsmäßig ruhigen Zeiten plötzlich wieder aktuell werden. Der »antitotalitäre Konsens « (Jürgen Habermas) ist daher heute und in künftigen Jahren so notwendig wie damals. Zu betonen ist, dass dieser Ordnungs-, Verfahrens- und Wertkonsens niemanden überfordern, dass er nicht den legitimen politischen Streit unzulässig eingrenzen will. Er gründet nicht in einer Idee der vorgegebenen Homogenität von Staat und Bürgern. Vielmehr will er - auf der Basis fundamentaler Gemeinsamkeiten - Streitaustrag in geordneten Formen möglich machen. Konsens und Konflikt stehen ja in einer Demokratie nicht in einem Gegensatz, sondern in einem Ergänzungsverhältnis. Das eine ist nicht ohne das andere denkbar. Es kann gefährlich sein, den politischen Streit auf die Spitze zu treiben, wenn man nicht über das Widerlager eines tragfähigen Fundamentalkonsenses verfügt. Aber nicht minder gefährlich kann es sein, den Austrag von Konflikten durch Homogenitätsforderungen zur Unzeit zu erschweren. Denn auch Streit, der nicht ausgetragen wird, kann das Gemeinwesen belasten.

(3) Ein weiterer Reformakzent im Bonner Grundgesetz war die Entscheidung für die repräsentative Demokratie, den »echten « Parlamentarismus und den Parteienstaat. Hier hat Bonn viele Weimarer Vorbehalte getilgt und viele Weimarer Rückzugsmöglichkeiten - etwa in einer Präsidialdemokratie - abgeschnitten. Die repräsentative Demokratie erscheint im Grundgesetz in reiner Form, ohne plebiszitäre Beimischungen und Vorbe-

15 Ernst Fraenkel, Gesammelte Schriften, hrsg. von Alexander von Brünneck u.a., Band 1: Recht und Politik in der Weimarer Republik, Baden-Baden 1999; ders., Deutschland und die westlichen Demokratien, erweiterte Ausgabe, Frankfurt a.M. 1991.

16 BVerfGE 2,1,12.

ZfP 56. Jg. 4/2009 
halte; dem Parlament werden nicht nur Öffentlichkeit und Diskussion als Aufgaben zugemutet, sondern auch Dezision, Verantwortung für die Regierung und ihre Stabilität und die Parteien werden ausdrücklich in die verfassungsmäßige Ordnung einbezogen.

Wie in anderen Ländern ist auch bei uns in den letzten Jahrzehnten das Parlament näher an die Regierung herangerückt. Es ist - den Prinzipien der Gewaltenteilung alten Stils zum Trotz - Teil des government geworden. Das gilt in doppelter Beziehung. Einmal steht das Parlament schon in seiner Funktion als Kreations- und Kontrollorgan der Exekutive in engerer Verbindung mit dem politischen Prozess als früher. Zum anderen werden aber auch seine Gesetzgebungsfunktionen vielfältig in die Zeitplanungen, in das Parteien- und Koalitionskalkül einer Regierung hineinverspannt. Als Sog zur Exekutive hin wirkt diese Entwicklung bis in die Personalstruktur der parlamentarischen Institutionen hinein, wobei sich nachdauernde Prioritäten der Staatsverwaltung mit modernen Tendenzen des »état actif « (Bertrand de Jouvenel) mischen.

Blickt man von heute her auf die Entwicklung von Parteiensystem und Parlament im Deutschland der Nachkriegszeit zurück, so zeigen sich charakteristische neue Mischungen. Manches erscheint undeutlicher als in den sechziger und siebziger Jahren. So hat sich die Konzentration und Stabilisierung des Parteiensystems nicht fortgesetzt, ${ }^{17}$ es ist kein Zweiparteiensystem entstanden wie in Großbritannien oder den USA - wohl auch wegen der strukturellen Grenzen des deutschen Wahlrechts. In der Entwicklung des Parlamentarismus ${ }^{18}$ liegen heute Tendenzen zur Gouvernementalisierung (nach britischem Vorbild) mit solchen zur Stärkung der parlamentarischen Autonomie im Widerstreit. Fundamentalalternativen zum parlamentarischen Regierungssystem - Rätedemokratie, Technokratie, Kybernetik - werden zwar kaum noch vertreten. Aber die Zahl derer, die einer stärker plebiszitären Ausgestaltung unserer Demokratie das Wort reden, ${ }^{19}$ ist unzweifelhaft gestiegen. Das zeigt, dass manche Gemeinsamkeiten in den Fundamenten heute nicht mehr so einfach vorausgesetzt werden kann wie 1949 oder noch 1969. Vor allem das repräsentative Element in unserem Regierungssystem bedürfte einer neuen werbenden Begründung.

(4) Von den Neuanfängen des Jahres 1949 scheint daher nur die Stärkung der Exekutive fast unbeschädigt die letzten Jahrzehnte überdauert zu haben. Hier ist der Unterschied zur Weimarer Demokratie mit ihren vergleichsweise schwachen und kurzlebigen Regierungen wohl am deutlichsten. Die »politische Gewalt« im Bonner System ist unabhängiger geworden. Für den Durchschnittsbürger ist heute zweifellos die Regierung - wenn nicht gar der Bundeskanzler, die Bundeskanzlerin - der wichtigste Bezugspunkt

17 Tilman Meyer / Volker Kronenberg (Hrsg.), Volksparteien: Erfolgsmodell für die Zukunft? Konzepte, Konkurrenzen und Konstellationen, Freiburg u.a. 2009; Franz Walter, Im Herbst der Volksparteien? Eine kleine Geschichte von Aufstieg und Rückgang politischer Massenintegration, Bielefeld 2009.

18 Heinrich Oberreuter / Uwe Kranenpohl / Martin Sebaldt (Hrsg.), Der Deutsche Bundestag im Wandel, Ergebnisse nenerer Parlamentarismusforschung, 2., durchgesehene und erweiterte Auflage, Wiesbaden 2002.

19 Hermann K. Heußner / Otmar Jung (Hrsg.), Mehr direkte Demokratie wagen. Volksentscheid und Bürgerentscheid: Geschichte, Praxis, Vorschläge, 2. völlig überarbeitete Auflage, München 2009. 
im Staat; der Bundestag wirkt manchmal - vor allem in den elektronischen Medien - eher wie ein Chor von Begleitstimmen. Wiederum liegen die britischen Parallelen klar zutage; sowohl das Premierminister-System wie die Vernetzung von Parlamentsmehrheit und Regierung kommen aus dem England des 19. und 20. Jahrhunderts.

Mustert man die »Kanzlerdemokratie «, ${ }^{20}$ so treten auch hier - in 60 Jahren und unter acht Kanzlern - Unterschiede der Form und des politischen Stils hervor. Regierte der patriarchalische Erzzivilist Adenauer sein Kabinett und seinen Staat mit straffer Hand und dürrem Wort, so führten Erhard und Kiesinger ihre Kabinette am längeren Zügel; zugleich wandten sie sich immer wieder mit appellativer Rhetorik an die Öffentlichkeit. Unter Kiesinger wurde das Amt des Kanzlers manchmal - koalitionsbedingt - zu einem »wandelnden Vermittlungsausschuss«; die Mühsal der Koordination überwucherte gelegentlich die Leitungsfunktion. Rhetorisch fordernd und appellativ war auch der Regierungsstil des ersten sozialdemokratischen Bundeskanzlers Willy Brandt; in seiner Amtszeit verselbständigte sich - ähnlich wie bei Kiesinger - der Apparat des Kanzleramtes unter einem eigenen Minister. Helmut Schmidt, aktenkundig und detailfreudig, holte diese Verselbständigung wieder ein, zog die Kabinettszügel straffer an und wandte sich oft mit pointierter Nüchternheit - Adenauer nicht unähnlich - an die Öffentlichkeit. Helmut Kobls Regierungsstil kombinierte Elemente seiner Vorgänger: Adenaners Generalistenart und demoskopische Neugier; Kiesingers und Brandts Gesprächigkeit, Schmidts Gedächtnis - neu war die Einbettung der zentralen Regierungsarbeit in Gruppendynamik: Politik nicht als Rechenaufgabe, sondern als Geländespiel. Gerhard Schröder setzte diesen Stil fort, teils als Medienkanzler mit populistischen Zügen, teils mit risikobereitem Blick in die Zukunft und gelegentlich erfrischendem »Basta«, während Angela Merkel bisher als Chefin einer Großen Koalition mit Kiesinger das Schicksal des »wandelnden Vermittlungsausschusses « teilte und allenfalls auf der internationalen Bühne größere Bewegungsfreiheit gewann.

Im Formwandel der Exekutive zeigen sich die Wandlungen der Zeit. In den Anfängen der Republik waren, nach Krieg und beispielloser Zerstörung, die Erwartungen bescheidener, die Chancen für Gehör und Gehorsam größer. Die Wiedererhebung des Landes aus der größten Katastrophe seiner neueren Geschichte, die Bewältigung der Kriegsfolgelasten, das Zusammenwachsen der Alt- und Neubürger, die sozialen und wirtschaftlichen Probleme - dies alles forderte und erlaubte einen strafferen Führungsstil. Heute würde sich eine Gestalt wie Adenauer unter den Westdeutschen schwerer tun als 1949: Seine Emotionslosigkeit würde leicht als mangelnde Sensibilität empfunden, seine Kühle als Unbetroffenheit, seine Ironie als Zynismus. Es regiert sich eben leichter im Klima kleiner persönlicher Hoffnungen als in einer Stimmung allgemeiner Ansprüche und des trotzigen Beharrens auf dem, »was mir zusteht «.

20 Karlheinz Niclauß, Kanzlerdemokratie. Regierungsfübrung von Konrad Adenauer bis Gerhard Schröder. Schöningh, Paderborn 2004. 


\section{III. »Abschied von der Größe»}

Hier ist daran zu erinnern, dass die geschilderten Entwicklungen - zum Rechtswegestaat, zur wehrhaften Demokratie, zur Parteiendemokratie und Kanzlerdemokratie-sich nicht allein in der politischen Zentrale, im »Treibhaus Bonn« und später in Berlin angebahnt haben; sie waren von Anfang an eingebettet in ein breiteres politisches Umfeld, in das Geflecht von Bund, Ländern und Gemeinden. Das bringt eine weitere Dimension des Grundgesetzes in den Blick: die bundesstaatliche Ordnung, den Föderalismus.

Deutschland war stets ein Reich, ein Bundesstaat oder Staatenbund, kurzum ein föderalistisches Gebilde. Zentralistische Perioden sind untypisch für unsere Geschichte. Andere Nationen erkennen und spiegeln sich in ihren Hauptstädten. In der deutschen Geschichte fehlt ein ähnliches, die politischen und kulturellen Kräfte sammelndes Zentrum, die Adressen der deutschen Hauptstädte wechseln durch die Geschichte hindurch - allein in neuerer Zeit lösen sich vier Hauptstädte ab: Wien und Frankfurt, Berlin und Bonn und seit den Neunzigerjahren wiederum Berlin. Die Zentralgewalt war in Deutschland fast immer schwächer als in anderen Staaten: Neben der Hauptstadt standen andere Zentren, und keine Stadt repräsentierte auf längere Zeit das ganze Deutschland - nicht politisch und schon gar nicht kulturell. Der Reichtum der Kultur in Deutschland nährt sich bis heute aus den Traditionen landesherrlichen Mäzenatentums. Abseits der großen Städte stößt man noch heute auf viele kleine Residenzen mit eigenem Gesicht - auf Theater, Konzertsäle, Archive, Bibliotheken, Kunstsammlungen hohen Ranges. Wolfenbüttel, Meißen, Hildburghausen, Weimar, Rothenburg haben sich stets neben Frankfurt, Köln, Berlin, München, Hamburg behauptet. Region war in Deutschland nie Provinz.

Nach 1949 hat das Parteiensystem dazu beigetragen, die föderalistische Ordnung zu stabilisieren. Bund, Länder und Gemeinden wurden zu gewichtigen, einander ergänzenden Rekrutierungs- und Aktionsfeldern der Parteien. Bis heute herrscht ein reger Austausch des politischen Personals hinüber wie herüber: Ein Bundeskanzler (oder ein Kanzlerkandidat) kommt in der Regel aus der Landespolitik, ein erfolgreicher Kommunalpolitiker kann ohne weiteres in die Landes- oder Bundespolitik wechseln - und umgekehrt. Die Wahlen auf den drei Gebietsebenen sind ein anstrengender, aber nützlicher Dauertest, der das politische System zwingt, sich dem Bürger gegenüber personell und programmatisch zu exponieren. Für den Zusammenhalt von Bund, Ländern und Gemeinden ist die durch Parteien verbürgte Homogenität ein Faktor der Stabilisierung.

Die föderalistische Struktur hat sich verändert in den 60 Jahren der Geltung des Grundgesetzes. Seit den sechziger Jahren hat man eine Zunahme unitarischer Tendenzen festgestellt, ausgelöst durch die wachsende Mobilität der Bevölkerung und das allmähliche Verblassen alter sozialer und landschaftlicher Zugehörigkeiten. ${ }^{21}$ Die Vereinheitlichung der Lebensformen, die Standardisierung von Produktion und Konsum, die Effizienz und Schnelligkeit moderner Kommunikationsmittel, die gleichmäßigen Ansprüche an Bildungsservice und sozialstaatliches »Netz«, kurz die Uniformität moderner Ge-

21 Konrad Hesse, Der unitarische Bundesstaat, Karlsruhe 1962. 
sellschaften - das alles war dem Föderalismus, seinem Aufbau in Schichten und Gliederungen, seinem komplizierten Geäst und Geflecht nicht günstig. So hat man denn in der Bundesrepublik Deutschland seit den sechziger Jahren den Föderalismus kooperativ "geöffnet«: Eine Reihe von Gemeinschaftsaufgaben zwischen Bund und Ländern wurde neu geschaffen, die Zuständigkeiten des Zentralstaats wurden verstärkt. Dies gilt selbst für den empfindlichen Bereich der Bildungspolitik - besonders für die Hochschulpolitik. ${ }^{22}$

Manche erwarteten damals, dass mit diesen Maßnahmen ein Prozess allmählichen Übergangs von Länderkompetenzen an den Bund beginnen werde. In der Tat haben sich die Gewichte in den siebziger Jahren zugunsten der Zentrale verschoben: Der Bund versuchte gelegentlich gegenüber den Ländern die Rolle des großen Bruders zu spielen. ${ }^{23}$ Doch ist inzwischen ein Umschwung eingetreten. Von einem »unitarischen Föderalismus « spricht man heute nur mehr in sehr gedämpftem Ton. Die vor Jahren prophezeite Stärkung der Zentralgewalt - erhofft von den einen, gefürchtet von den anderen - ist nicht eingetreten. Der Föderalismus hat sich in seiner überlieferten Form behauptet; ja, er wurde noch gestärkt durch die 1992 im Hinblick auf den europäischen Integrationsprozess vorgenommenen Verfassungsänderungen und durch die beiden Reformkommissionen aus jüngster $\mathrm{Zeit}^{24}$ - wenn er auch überwiegend die Gestalt eines Exekutivföderalismus auf Kosten der Landesparlamente angenommen hat.

Das fällt zusammen mit einem Wandel der Mentalitäten. Nach Jahren einer fast rauschhaft erlebten Mobilität macht sich heute ein stärkeres Verlangen nach Befestigung, Überschaubarkeit, stabilen Zuordnungen geltend. Auch ein größerer Realismus: Man will wissen, wo man dran ist, wie man die Politik »vor Ort« einzuschätzen hat. Traditionen, Landesprofile, Lebensgewohnheiten werden neu entdeckt. Standorte spielen eine Rolle, Qualitäten des Angebots. Hat ein Land gute Schulen, eine gute Wirtschafts- und Arbeitspolitik? Kurzum, das Überschaubare ist gefragt. Man misstraut großsprecherischen Ankündigungen aus der Ferne. Der Föderalismus als Element des Wettbewerbs, des Vergleichs, der Probe aufs Exempel - das dürfte sich in einem Europa, in dem sich das globalisierte Allgemeine an jedem Fleck in regionalen Überlieferungen bricht, noch verstärken.

Die doppelt - rechtsstaatlich und föderalistisch - gezähmte Bonner Demokratie ist eingeschworenen Anhängern kontinentaler Staatsüberlieferungen immer als ein unfertiger Staat erschienen. So hat General de Gaulle im Gespräch mit Adenauer Zweifel daran geäußert, ob ein so ausgeprägt föderalistisches Gemeinwesen wie die Bundesrepublik

22 Fritz W. Scharpf / Bernd Reissert / Fritz Schnabel, Politikverflechtung: Theorie und Empirie des kooperativen Föderalismus in der Bundesrepublik, 2 Bände, Kronberg 1975.

23 Vgl. etwa Ursula Münch, Sozialpolitik und Föderalismus. Zur Dynamik der Aufgabenverteilung im sozialen Bundesstaat, Opladen 1997.

24 Siehe auch zu den jüngsten Reformen Rainer Holtschneider / Walter Schön (Hrsg.), Die Reform des Bundesstaates. Beiträge zur Arbeit der Kommission zur Modernisierung der bundesstaatlichen Ordnung 2003/2004 und bis zum Abschluss des Gesetzgebungsverfabrens 2006, Baden-Baden 2007; Beschlüsse der Kommission zur Modernisierung der bundesstaatlichen Ordnung bzw. zur Modernisierung der Bund-Länder-Finanzbeziehungen, Kommissionsdrucksache 174 (2009). 
Deutschland - noch dazu mit ausgebauter Verfassungsgerichtsbarkeit - überhaupt noch ein Staat im klassischen Sinne sei. Unverkennbar ist der Staat des Grundgesetzes ein bescheideneres Gebilde als die Weimarer Republik. Die Tendenz zur Beschränkung, zur Minimalisierung der Staatsmacht ist nicht zu übersehen. »Erlösung von der Größe« hat Fritz Stern das genannt, ${ }^{25}$ von »Verschweizerung « sprach Alfred Grosser, ${ }^{26}$ von »Machtvergessenheit « Hans-Peter-Schwarz. ${ }^{27}$ Und in der Tat: Nie waren die Deutschen so bereit, Rücksicht zu nehmen, blindes Auftrumpfen zu vermeiden, sich an Regeln des politischen Spiels zu binden, wie in der Bonner Demokratie. Dieser Staat war kein ungestümer Täter mehr, nicht mehr der sprichwörtliche Elefant im Porzellanladen - er bewegte sich im Regelfall anmutig nach Rechtsfiguren. Nach draußen entwickelte er eine Diskretion, die keineswegs gespielt und vorgeschützt war. Vor weltpolitischen Engagements hielt er sich lange Zeit vorsichtig zurück. Auch im Inneren beachtete er Bescheidenheitsrituale: Das Prinzip der Bürgernähe verlangte, dass der Staat behutsam auftrat und keinen Anspruch machte auf den Nimbus des Besonderen und Hervorgehobenen. Und das war - nach den Exzessen des Auftrumpfens und der Selbstüberschätzung im Dritten Reich - gewiss so übel nicht.

Der verminderte Zuschnitt der politischen Gewalt hat übrigens den wirtschaftlichen Erfolg der Bonner Republik nicht gehindert. Ganz im Gegenteil: Der Verzicht auf übermäßigen politischen Ehrgeiz kam der Entfaltung wirtschaftlicher Aktivität, dem Gedeihen von Produktion und Konsum zugute. Im Rahmen einer Staatlichkeit mit niederem Profil konnten sich die alten bürgerlichen Tugenden des Fleißes, der genossenschaftlichen Kooperation, des Managements und der Organisation voll entfalten. Früh waren Lebensmittelkarten und Zwangswirtschaft gefallen, ein freier Markt hatte sich entwickelt, die aus der Kriegszeit ererbten Notstände lösten sich auf. Die große Umverteilung zwischen Heimatvertriebenen und Ansässigen, der Lastenausgleich, wurde möglich. Ludwig Erhard - bis heute der einzige wirkliche Systemveränderer in unserer Nachkriegsgeschichte - brachte die versteinerten gesellschaftlichen Verhältnisse zum Tanzen. Aus den besitzlosen »Normalverbrauchern« wurde ein Volk, das wachsenden Wohlstand errang, ein Volk von Reisenden und Autobesitzern. Wohneigentum und Hausbesitz entwickelten sich; das Wort » Wirtschaftswunder « machte die Runde. Es fand Eingang auch in andere Sprachen. Dabei übernahm Deutschland in seiner Wirtschaftspolitik nie die reine Lehre des Liberalismus: Die Freisetzung wirtschaftlicher Energien wurde wirksam ergänzt durch Elemente der Ordnung, der sozialen Balance ${ }^{28}$ - ein System, für das sich verschiedene Namen einbürgerten: »Ordo-Liberalismus«, "soziale Marktwirtschaft «, »rheinischer Kapitalismus«.

25 Fritz Stern, Der Traum vom Frieden und die Versuchung der Macht. Deutsche Geschichte im 20. Jabrhundert, Berlin 1999, Kap. III.

26 Alfred Grosser, Die Bonner Demokratie. Deutschland von draußen gesehen, Düsseldorf 1960.

27 Hans-Peter Schwarz, Von der Machtbesessenbeit zur Machtvergessenheit, München 1985.

28 Wilhelm Röpke, Jenseits von Angebot und Nachfrage, 5. Auflage, Bern 1979. 
An dieser Stelle ist an die gewaltige praktische Entfaltung des Sozialstaats zu erinnern - gewiss eine der wichtigsten Konkretisierungen der Verfassung überhaupt. ${ }^{29}$ Nur an zwei Stellen spricht das Grundgesetz zusammenfassend vom »sozialen Staat « - die Ausgestaltung im einzelnen wurde dem politischen Prozess überlassen, wobei von Anfang an die Rechtsprechung eine wichtige Rolle spielte. Die Tarifautonomie entlastete den Staat von einer Sisyphus-Aufgabe. Die sozialen Sicherungen wurden ausgebaut - so stark und dicht, dass heute nicht mehr nur die unmittelbare Notbewältigung im Vordergrund steht, sondern zugleich - als »Sozialpolitik zweiter Ordnung (Franz Xaver Kaufmann) - die Aufgabe, bereits bestehende Leistungssysteme funktionsfähig zu erhalten und die von ihnen ausgelösten Wechselwirkungen zu bewältigen. Auch der Sozialstaat ist in Deutschland mit einer gewissen Grundsätzlichkeit hervorgetreten, als stabilisierende, reformistische Reaktion auf Defizite des Liberalismus - heute steckt er in einer kritischen Situation, weil verschiedene Herausforderungen gleichzeitig zusammentreffen: die Alterung der Gesellschaft, die Umbrüche im Arbeitsmarkt, die weltweiten Wirkungen der Globalisierung, die aktuelle Finanz- und Wirtschaftskrise.

\section{Das vereinigte Deutschland und die europäische Zukunft}

Im Lauf seiner 60jährigen Geschichte musste sich das Grundgesetz in unterschiedlichen Situationen bewähren. Es musste auf technische Veränderungen reagieren, von der Luftfahrt bis zu den elektronischen Medien, es musste politische Lücken schließen, von der Landesverteidigung bis zur Regelung des Notstands, es musste sich öffentlich diskutierten neuen Fragen stellen: dem Umweltschutz, den Problemen der Behinderten, der Durchsetzung der Gleichberechtigung, dem Verbot von Diskriminierungen und nicht zuletzt den innerstaatlichen Wirkungen der europäischen Integration. ${ }^{30}$ Dass das Gefüge der Bundesrepublik Deutschland in vielfältiger Weise offen war für Kooperation, für europäische und weltweite Zusammenarbeit, erwies sich als Stimulans für die Zukunft. Ein abgeschlossenes, fertiges staatliches Gebilde war die Zweite Republik nie: Einmal hatte sie von Anfang an unter dem Gebot der Wiedervereinigung Rücksicht auf »Deutschland als Ganzes « zu nehmen, sodann hatte sie in Art. 24 ausdrücklich für die internationale Zusammenarbeit optiert - eine Option, die seither in vielen Dimensionen, bilateral und multilateral, realisiert wurde. Von der Kontrollrats-Zeit bis zur Gegenwart stand und steht Deutschland in einer Fülle externer Verbindungen und Abhängigkeiten, in einer komplexen Interaktion mit vielen Räumen der Welt, vom Europa des Europarats, davor der Europäischen Gemeinschaft, der Europäischen Union bis zur transatlantischen Gemeinschaft der westlichen Welt; von der Vielzahl der Entwicklungsländer bis zu den Transformationsländern des Postsozialismus. So haben im Lauf der Zeit auch zahlreiche

29 Stephan Leibfried / Uwe Wagschal (Hrsg.), Der deutsche Sozialstaat. Bilanzen - ReformenPerspektiven, Frankfurt a.M. 2000.

30 Roland Sturm / Heinrich Pehle, Das neue deutsche Regierungssystem. Die Europäisierung von Institutionen, Entscheidungsprozessen und Politikfeldern in der Bundesrepublik Deutschland, 2. aktualisierte und erweiterte Auflage, Wiesbaden 2006. 
Ausländer Zugang zur deutschen »Inlandsgesellschaft« gefunden, sei es im Rahmen der europäischen Integration, sei es in vielfältigen Arbeitsverhältnissen, sei es im Strom der Menschen, die das Asylrecht für politisch Verfolgte in Anspruch nahmen oder sonst wie in Deutschland Zuflucht vor Krieg und Not suchten.

Zum eindrucksvollsten Test auf die Anpassungsfähigkeit und Integrationskraft des Grundgesetzes wurde die Wiedervereinigung. Die größte Veränderung in der deutschen Geschichte nach 1949 ging so vor sich, dass diejenigen, denen 1949 »mitzuwirken versagt war «, 40 Jahre später in Gestalt der »neuen Länder« die Ordnung des Grundgesetzes übernahmen - womit der alte Artikel 23 im doppelten Sinn des Wortes »aufgehoben« war und im Vertragsschluss (des Einigungsvertrages) »sterben « konnte. ${ }^{31}$ Damit ging ein großer Teil der Provisorien und »Transitorien« der Nachkriegszeit zu Ende, während das Grundgesetz - nunmehr zur Ordnung des ganzen Deutschland geworden - weiterbestand: ein Rechtskontinuum, das die alte und die neue Bundesrepublik eindrucksvoll miteinander verklammert.

Deutschland verfügte nun über feste Grenzen, völkerrechtliche Souveränität und einen Platz in einer europäischen Ordnung. Es erhielt im 20. und 21. Jahrhundert, nach einem unsäglichen Absturz, seine "zweite Chance« (Fritz Stern). Den ost-westlichen Dualismus, der Deutschland 40 Jahre lang in zwei politischen Gestalten existieren ließ, gab es plötzlich nicht mehr (1). Die Deutschen fanden sich nach 1990 in einem »Europa der Vaterländer « wieder (im Osten nicht minder als im Westen, Süden und Norden!) - sie mussten also nun ihr Nationalinteresse ebenso umschreiben, wie dies die Franzosen, die Italiener, die Polen, die Ungarn und andere Völker seit jeher taten, damit das verbindende europäische Interesse deutlicher hervortreten konnte (2). Und endlich erwarteten die europäischen Nachbarn von den Deutschen so etwas wie eine berechenbare Normalität - sie erwarteten, dass die Deutschen verlässliche Traditionen und Verhaltensmuster entwickelten und dass sie mit sich selbst ins Reine kamen (3).

(1) Es war nach 1945 nicht immer leicht, ein Deutscher zu sein. Vor der Weltöffentlichkeit standen die Deutschen, stand die ganze deutsche Geschichte am Pranger - nicht nur die jüngste, sondern auch die weiter zurückliegende Vergangenheit. So haben vor allem in der alten Bundesrepublik viele Deutsche in bester Absicht ihre humanistischen Überzeugungen, ihren europäischen und weltbürgerlichen Part betont. Das führte freilich manchmal zu seltsamen Flucht- und Absetzbewegungen von der deutschen Nation und ihrer so belasteten und belastenden Geschichte. Einen grotesken Fall erzählt Andrzej Szczypiorski: »Ich war bei netten Leuten in Baden-Baden zu Gast. Eines Tages sagte mein Gastgeber zu mir: 'Wissen Sie, wir Badener sind eigentlich keine Deutschen. Blicken Sie von der Terrasse aus nach Westen. Dort, wo Sie diese Wiesen und Weinberge sehen, ist schon Frankreich. Wir haben lateinische Gemüter, lieben Wein, schöne Frauen, Liebeslieder. ... Einige Tage später war ich in Hamburg. Und dort hat mir ein netter Mann in einer Tweedjacke, die Pfeife im Mund, gesagt: Wissen Sie, wir Hamburger sind eigentlich keine Deutschen. Wir haben eine hanseatische Tradition, skandinavische Sitten. Wir halten uns nicht für die Deutschen. Ich erzählte ihm dann von meinem Gespräch in Baden-

31 Wolfgang Schäuble, Der Vertrag. Wie ich über die deutsche Einheit verhandelte, Stuttgart 1991. 
Baden und fragte: , Wenn es wirklich wahr ist, dass weder die Badener noch die Hamburger Deutsche sind, sagen Sie mir bitte, wo die Deutschen leben.< Er entgegnete mir sehr ruhig: >Fahren Sie in die DDR. Dort leben die Deutschen.« ${ }^{32}$

Solche Szenen sind nach der Vereinigung nicht mehr so leicht vorstellbar. Die Neigung zur politischen Mimikry hat abgenommen. Politische Schutzfarben für Deutsche sollten heute nicht mehr nötig sein, obwohl die Liebe zu Deutschland und den Deutschen in den letzten Jahren gewiss nicht größer geworden ist - bei anderen Völkern nicht und nicht einmal bei uns selbst. Aber ein wenig Realismus hat sich doch ausgebreitet: Die Deutschen sind eher bereit, einander so zu nehmen, wie sie sind - und auch Deutschlands Nachbarn erwarten keine neuen spektakulären Wandlungen (die ja auch ihre Risiken hätten). So ist nach 1990 ein Stück Normalisierung erreicht worden: Der Kalte Krieg ist zu Ende, die Deutschen können ihre bipolaren Unterstände verlassen - sie stehen freilich jetzt genauso im Freien, in Sonne und Regen, wie andere Völker auch.

(2) Die Deutschen leben inmitten von Nationen und Nationalstaaten. Kein Nachbar in den vier Himmelsrichtungen, der nicht auch Deutschland als Nation, als Nationalstaat sieht. Verstehen sich die Deutschen anders, so muss das gerade auf unsere mittel-, ostund südosteuropäischen Nachbarn verwirrend und irritierend wirken; denn sie haben sich ja gegenüber dem sowjetischen Imperialismus gerade kraft ihrer nationalen Identität behauptet. Was hatten unterdrückte Völker von den Polen bis zu den Ukrainern, von den Ungarn bis zu den Albanern, von den Esten bis zu den Tschechen und Slowaken der östlichen Hegemonialmacht anderes entgegenzusetzen als ihre eigene Geschichte? Worte wie Heimat, Vaterland, Nation, Patriotismus haben daher in diesen Ländern auch heute einen guten Klang. Keines dieser Völker kann sich die Zukunft ohne Nationalstaat vorstellen. Begegnet ihnen im Westen ein Deutschland, das nicht Nationalstaat sein will, so wird es leicht als potentielle Hegemonialmacht identifiziert - als ein auf Expansion angelegtes Gebilde, das sich - bewusst oder unbewusst - der Selbstbeschränkung eines Nationalstaats mit klar definierten Interessen entziehen will.

Das schließt selbstverständlich nicht aus, dass die Deutschen, belehrt durch ihre jüngste Geschichte, aus ihren europäischen und atlantischen Erfahrungen heraus ein offenes Konzept des Nationalstaats vertreten, mit Minderheitenschutz und Minderheitenrechten, mit einer föderalistischen Staatsorganisation, die das Eigenleben der Regionen stärkt, mit der Bereitschaft zur internationalen Zusammenarbeit und zur übernationalen Integration. Das entspricht der Entwicklung, die sich in West-, Süd- und Nordeuropa seit 1951 vollzogen hat.

Hier sind alte nationale Rivalitäten zurückgedrängt und abgebaut worden durch das Programm europäischer Kooperation und Integration. Dadurch ist vor allem der alte Konflikt zwischen Deutschland und Frankreich entschärft und das französisch-deutsche Verhältnis zu einer Antriebskraft für Europa gemacht worden.

Die Konzepte von Nationalstaat und übernationaler Integration lassen sich über eine weite historische Strecke hin verbinden, wie die Entwicklung vom Schuman-Plan zu den Verträgen von Maastricht, Amsterdam, Nizza und schließlich Lissabon zeigt. In der

32 Andrzej Szczypiorski, Reden über Deutschland, München 1990, S. 94 f.

ZfP 56. Jg. 4/2009 
»nachholenden Integration« des europäischen Ostens dürfte der Nationalstaat jedenfalls noch längere Zeit eine Rolle spielen, obwohl seine Öffnung im eben beschriebenen Sinn schon heute beginnen kann - und auch beginnen sollte. Das Ziel muss, in West- wie Osteuropa, dasselbe sein: eine Ordnung, in der Konflikte durch Zusammenarbeit entschärft werden, gemeinsame Interessen zwischen den Völkern entstehen, Kriege undenkbar werden.

(3) Am schwierigsten wird es sein, der künftigen deutschen Geschichte so etwas wie Normalität zurückzugeben und nach den Exzessen der NS-Despotie Raum für einen schlichten Patriotismus, ein bescheidenes Nationalgefühl zu schaffen. Wer das versucht (und ich halte es gerade im Interesse unserer Nachbarn für notwendig), der muss sich mit der Tatsache auseinandersetzen, dass Hitler und sein Regime nicht nur gekennzeichnet waren durch Extremismus und Maßlosigkeit und unsagbare Verbrechen. Eine der bösesten Hinterlassenschaften des Nationalsozialismus ist der Missbrauch und die ibm folgende Diskreditierung des Normalen. Hitler errang ja seine Erfolge nicht allein mit Drohungen und Terror, er beutete auch Gefühle aus, Opfersinn, Anhänglichkeit der Massen, Begeisterung der Jugend. Auch die Vaterlandsliebe ist im Dritten Reich schauerlich missbraucht worden, daran ist kein Zweifel. So war alle Art von Patriotismus unter den Deutschen nach dem Krieg gründlich verpönt und verrufen - und eilfertig verbrannten viele, was sie vorher angebetet hatten. Heute ist, wie Umfragen bis in die jüngste Zeit hinein zeigen, nur eine Minderheit der Deutschen auf ihre Heimat stolz, nicht wenige stehen der wiedervereinigten Nation skeptisch oder ablehnend gegenüber, und bei internationalen Schülertreffen erkennt man die jungen Deutschen oft daran, dass sie die einzigen sind, die ihre Nationalhymne nicht singen können.

Doch das undifferenzierte Wüten gegen alles, was das Dritte Reich einmal missbraucht hat, kann am Ende Hitlers böse Hinterlassenschaft nicht wirklich überwinden. Gegen eine so einseitige Therapie, die ohne Unterscheidung und Differenzierung verfährt, bauen sich nur Resistentien auf: Gleichgültigkeit, Verstocktheit, Trotz. Wer mit dem von Hitler Missbrauchten auch das Normale, Alltägliche verwirft, das alten Demokratien ganz selbstverständlich zu eigen ist, der unterliegt einem Fehlschluss, über den gerade Hitler grimmige Genugtuung empfinden müsste - er, der seinem Volk im Führerbunker 1945 bekanntlich den Untergang gewünscht hat. Falsche Antithesen zu Hitler könnten daher leicht zu Hitlers späten Siegen werden.

Dies gilt besonders für den Umgang mit Nation und Vaterland. Wer meint, die mögliche Wiederkehr des Nationalsozialismus am besten dadurch zu verhindern, dass er »Nie wieder Deutschland! « ruft, der unterliegt einer ebenso naiven wie gefährlichen Illusion. Wer Nation und Patriotismus tabuisieren will, der bewirkt höchstens, dass beides in die Hände von Extremisten fällt. Tabuisierungen und Sprechverbote sind im Allgemeinen von sehr wenigen Ausnahmen abgesehen - keine vernünftige Politik. Ein aufgeklärter Patriotismus wird vielmehr den Dialog suchen, und er wird alles daran setzen, die von der Nazi-Tyrannei missbrauchten Loyalitäten der Bürger gegenüber dem Staat für den 
alläglichen Politik-Gebrauch zurückzufordern. Denn ohne diese bürgerliche Normalität kann keine Demokratie existieren und gedeihen. ${ }^{33}$

Das heißt nicht, dass wir unser Land mit blindem Stolz bewundern und es lieben fast um jeden Preis. Zurückhaltung, Skepsis, Selbstkritik sind angebracht nach all dem, was in den Nazijahren im deutschen Namen geschah. Aber es gibt auch die Chance eines neuen Anfangs. Man kann Lehren aus der Geschichte ziehen. Das Grundgesetz ist eine solche Lehre. Es hat sich in 60 Jahren als Charta unserer neugewonnenen Freiheit, als »Gedächtnis der Demokratie « ${ }^{34}$ bewährt. Indem es die Macht des Staates begrenzte und die Rechte des Bürgers sichern half, indem es das Nicht-Strittige, den Wertkonsens, hervorhob und damit dem politisch-parlamentarischen Kampf einen festen Rahmen gab, hat es der Demokratie in Deutschland eine neue Chance eröffnet. Mit ihm verbindet sich die Hoffnung, dass auch aus einer scheinbar aussichtslosen Situation, aus einer verworrenen und belasteten Geschichte Wege in die Zukunft führen.

\section{Zusammenfassung}

Die Ausgangsbedingungen der Bundesrepublik vor 60 Jahren waren nicht einfach: Sie musste die Folgen des Krieges, die Erblasten des Nationalsozialismus und die Teilung Deutschlands im heraufziehenden Kalten Krieg verarbeiten. Auf diesem Hintergrund stellt das Grundgesetz von 1949 einen bemerkenswerten Neubeginn dar. Es zog mit dem konsequenten Rechtsstaat, dem Prinzip der wehrhaften Demokratie, dem Bekenntnis zum parlamentarischen Regierungssystem und der Stärkung der Exekutive wichtige Lehren aus dem Scheitern der Weimarer Republik. Diese Lehren sollten sich als Basis für den Erfolg der Bundesrepublik erweisen. Der historisch verankerte Föderalismus schützte dabei vor einer allzu mächtigen Zentralgewalt. Das Ende des Kalten Krieges und die europäische Integration stellen neue Herausforderungen an das wiedervereinigte Deutschland: Es gilt ein neues gesamtdeutsches Selbstverständnis zu finden, das Normalität und Nationalbewusstsein mit dem schweren Erbe der deutschen Geschichte in Einklang bringt.

\section{Summary}

The initial conditions for the Federal Republic of Germany were not easy: Germans had to face the outcome of World War II., the heritage of National Socialism and the fact of a splitted Germany in the just started Cold War. With this background, the German Grundgesetz was a really notable chance for a new beginning. Principles like the strong state of law, the militant democracy and the strengthening of the executive show that the German Constitution has learned its lessons from the disaster of the Republic of Weimar.

33 Dazu Volker Kronenberg, Patriotismus in Deutschland. Perspektiven für eine weltoffene Nation, 2. Auflage, Wiesbaden 2006.

34 Paul Kirchhof, »Das Grundgesetz als Gedächtnis der Demokratie« in: Martin Heckel (Hrsg.), Die innere Einheit Deutschlands inmitten der europäischen Einigung, Tübingen 1996, S. 35-51.

ZfP 56. Jg. 4/2009 
History until now shows that these elements were the best basis for the success of the Federal Republic. In addition, the historically rooted federal organisation protects against a too powerful central authority. The end of the Cold War and the European integration are new challenges for the reunified Germany: it needs a new self-perception which combines the difficulties based in the German history and a kind of normality for the German people.

Hans Maier, A successful provisorium. The Grundgesetz after 60 years

\section{Politika. Passauer Studien zur Politikwissenschaft}

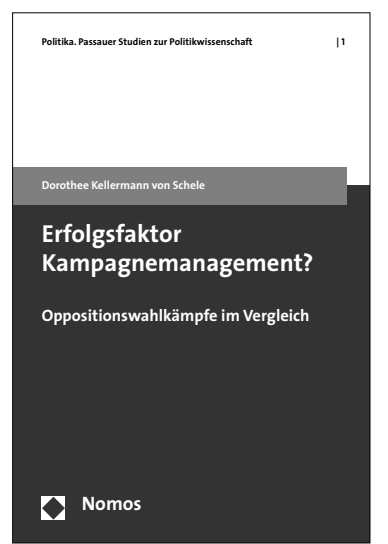

Bitte bestellen Sie im Buchhandel oder versandkostenfrei unter www.nomos-shop.de

\section{Erfolgsfaktor}

Kampagnemanagement?

Oppositionswahlkämpfe im Vergleich

Von Dorothee Kellermann von Schele

2009, Band 1, 272 S., brosch., 29, - $€$,

ISBN 978-3-8329-4892-4

Der Wahlkampf der SPD 1998 gilt als gelungenes Beispiel einer konsequent vorbereiteten und umgesetzten Wahlkampagne. Warum ist es der Union mit ihrem Kampagnemanagement trotz günstiger Umfragedaten im Vorfeld der Wahl weder 2002 noch 2005 gelungen, an den Erfolg der Kampagne anzuknüpfen? Wo liegen Restriktionen, wo Erfolgsfaktoren für professionelle Wahlkampfführung? 